\title{
[Letter]
}

\section{Supercritical Phase Alkylation Reaction on Solid Acid Catalysts}

Ikusei Nakamura, Li Fan*, Shintaro Ishida and Kaoru Fujimoto

Department of Applied Chemistry, School of Engineering

The University of Tokyo, Hongo, 113, Bunkyo-ku, Tokyo, Japan

(Received September 5, 1996)

\begin{abstract}
Alkylation reaction on zeolite catalysts was conducted in supercriticalphase 2-methyl-propane which was reactant as well. Compared to the reactions conducted in liquid phase or gas phase, the supercritical phase reaction exhibited higher activity, along with obviously longer lifetime, on Ytype zeolite catalyst. Supercritical fluid was able to extract in situ and transport high-molecular-weight olefins to extend catalyst life successively.
\end{abstract}

\section{Introduction}

Due to the Clean Air Act, increasing attention is paid to production method of alkylate, which is a very clean burning fuel and has a high MON (motor octane number) with a low octane sensitivity and moderate vapor pressure. Commercially-operated process of alkylate production uses liquid acid catalyst such as $\mathrm{H}_{2} \mathrm{SO}_{4}$ or $\mathrm{HF}$, resulting in a lot of problems in cost, apparatus and environment "). New synthesis method utilizing solid acid catalysts has been developed for a long time but no commercial process is realized due to the fast catalyst deactivation ${ }^{2}$.

Application of supercritical fluid in heterogeneous catalysis is of great interest recently ${ }^{31}$. Selected supercritical fluid was demonstrated to be able to extract high-molecular-weight hydrocarbons from catalyst micropores, to improve catalyst deactivation ${ }^{4), 5)}$ or to reactivate spent catalyst ${ }^{6}$. Here we applied supercritical fluid to alkylation reactions on solid acid catalysts and investigate the catalytic performances of this process.

\section{Experimental}

Commercially available H-USY (Catalyst $\&$ Chemical Ind., $\mathrm{SiO}_{2} / \mathrm{Al}_{2} \mathrm{O}_{3}=8.6$ ) catalyst was mainly utilized with the pellet of 20-40 mesh. Before reaction it was calcined in flowing air for $3 \mathrm{~h}$ at $450^{\circ} \mathrm{C}$ in situ ${ }^{7}$. A continous flow type fixed-bed reactor equipped with upstream preheater was utilized. Tailor-made reactant mixture with different composition (olefin/paraffin ratio varied between 1/5 and 1/70) was pressurized into the high pressure pump before reaction. The products were determined by GC-MASS

* To whom correspondence should be addressed. 
(Shimadzu GCMS QP1100EX). Quantitative analysis was conducted by on-line gas-chromatography. Reaction conditions were: reaction temperature, $140^{\circ} \mathrm{C}$; reaction pressure, 60atm; 2-methyl-propene/2methyl-propane ratio $=1 / 50 ; \mathrm{W} / \mathrm{F}($ olefin $)=40 \mathrm{~g} \cdot \mathrm{h} / \mathrm{mol}$.

The critical pressures and critical temperatures of the fluids used were listed here: propane, $96.8^{\circ} \mathrm{C}$ and 42atm; 2-methyl-propane, $135^{\circ} \mathrm{C}$ and $36 \mathrm{~atm} ; 2-$-methyl-butane, $188^{\circ} \mathrm{C}$ and 33atm. The paraffins acted here as both reactant and supercritical fluid, except propane.

\section{Results and Discussion}

Fig. 1 compared the durability results of the 2-methyl-propene and 2-methyl-propane reaction at gas phase, liquid phase and supercritical phase, respectively. The catalyst was calcined at $450^{\circ} \mathrm{C}$. Very high initial activity, where the yield of alkylate $(2,2,4$,trimethylpentane) was as high as $70 \%$, appeared at the liquid-phase reaction $\left(50^{\circ} \mathrm{C}, 35\right.$ atm). But no activity was observed when the accumulated feed amount of olefin reached 20 $\mathrm{mmol} /$ cat.g. Similarly, obvious catalyst deactivation was observed at gas-phase reaction. Alkylate yield dropped to near zero at the accumulated olefin feed amount of 20 $\mathrm{mmol} / \mathrm{cat} . \mathrm{g}$. But for the supercritical-phase reaction, the catalyst deactivation was suppressed by supercritical fluid. While the

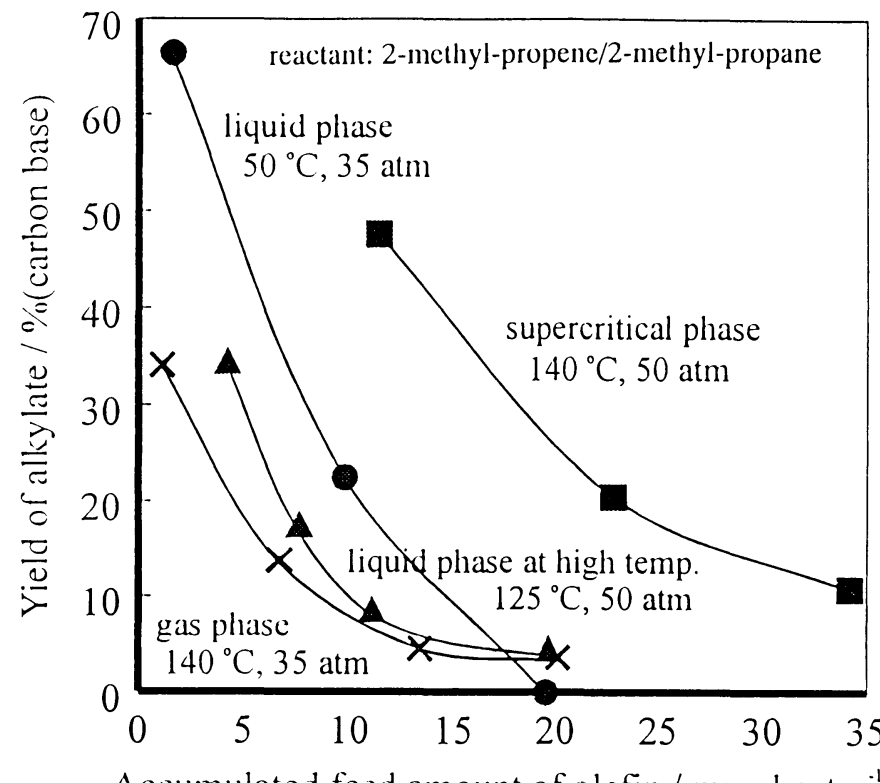

Accumulated feed amount of olefin $/ \mathrm{mmol} \cdot \mathrm{cat}^{-\mathrm{g}^{-1}}$

Fig.1 Reaction Phase Effect on H-USY

(standard reaction conditions) accumulated olefin feed amount reached $35 \mathrm{mmol} / \mathrm{cat} . \mathrm{g}(5.6 \mathrm{~h})$, alkylate yield was still higher than $10 \%$. Although the yield of alkylate decreased with time-on-stream, 2-methyl-propene conversion was almost $100 \%$. For another liquid phase reaction whose reaction conditions $\left(125^{\circ} \mathrm{C}, 50 \mathrm{~atm}\right)$ were slightly different from those of supercritical phase reactions, it exhibited similar deactivation behavior to that of gas phase reaction.

It should be mentioned that supercritical-phase and gas-phase reactions were implemented at $140^{\circ} \mathrm{C}$, but liquid-phase reactions were conducted at $50^{\circ} \mathrm{C}$ or $125^{\circ} \mathrm{C}$. For the gas-phase reaction, high reaction temperature favored side-reactions and secondary reactions, deactivating the catalyst easily. Consequently the initial alkylate yield was only about $30 \%$ for the gas-phase reaction, rather lower than that for the supercritical-phase reaction. C5-C7 hydrocarbons formed in relatively high selectivity in gas- or supercritical-phase reaction, which should be attributed to the high reaction temperature in either case. In the liquid-phase reaction $\left(50^{\circ} \mathrm{C}\right), \mathrm{C} 12$ olefin formed in high selectivity, especially after the catalyst deactivation. This can be reasoned to the slow diffusion of the reactant and produced olefin oligomer inside liquid phase reaction medium. The residence time of the olefins would be prolonged in the catalyst bed at 
the liquid-phase reaction, compared to that at the supercritical-phase reaction ${ }^{5}$.

Supercritical fluid exhibits quite different extraction capacity if their molecular weight is changed. In Fig. 2, it is investigated the durability of alkylation reaction activity where 2-methyl-butane, 2methyl-propane and propane at the supercritical phase was utilized as the reaction medium, respectively. It should be mentioned that 2-methyl-butane and 2methyl-propane were reactants as well. For the 2-methyl-butane case, deactivation of the alkylate yield was hardly observed, exhibiting low initial yield as to $20 \%$. On the contrary, high initial yield of about $60 \%$ was reached in the reaction implemented in supercritical phase propane while deactivation was most obvious among these. The reaction conducted in 2-methyl-propane showed the moderate results, compared to the reactions in 2-methyl-butane or propane.

The reason for the low alkylate yield in 2-methyl-butane reaction should be its high reaction temperature. High reaction temperature favored the side reactions, reducing the selectivity of alkylate. Indeed, C5-C7 hydrocarbon products formed in high selectivity in the high-temperature-reaction as conducted in supercritical phase 2methyl-butane. It is possible that hydrocracking reaction, a side reaction, occurred at the same site where alkylation reaction proceeded. The reaction temperature of the propane-used reaction was low and the side reactions were effectively suppressed. The deactivation of this reaction should be due to the poor extraction capacity of propane medium,

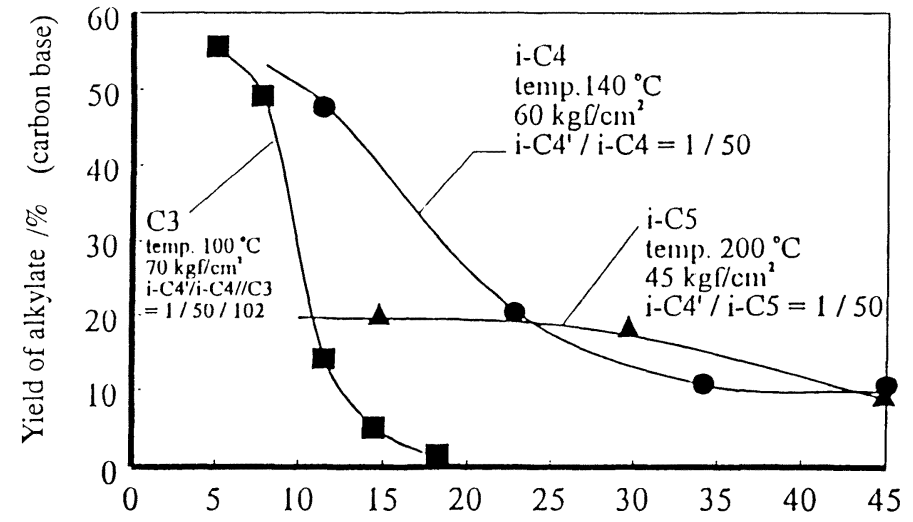

Accumulated feed amount of olefin $/ \mathrm{mmol}$ cat-g ${ }^{-1}$

Fig. 2 Reaction in Different Supercritical Fluid

(standard reaction conditions; i-C4':2-methyl-propene; i-C4:2-methyl-propane; i-C5:2-methyl-butane)

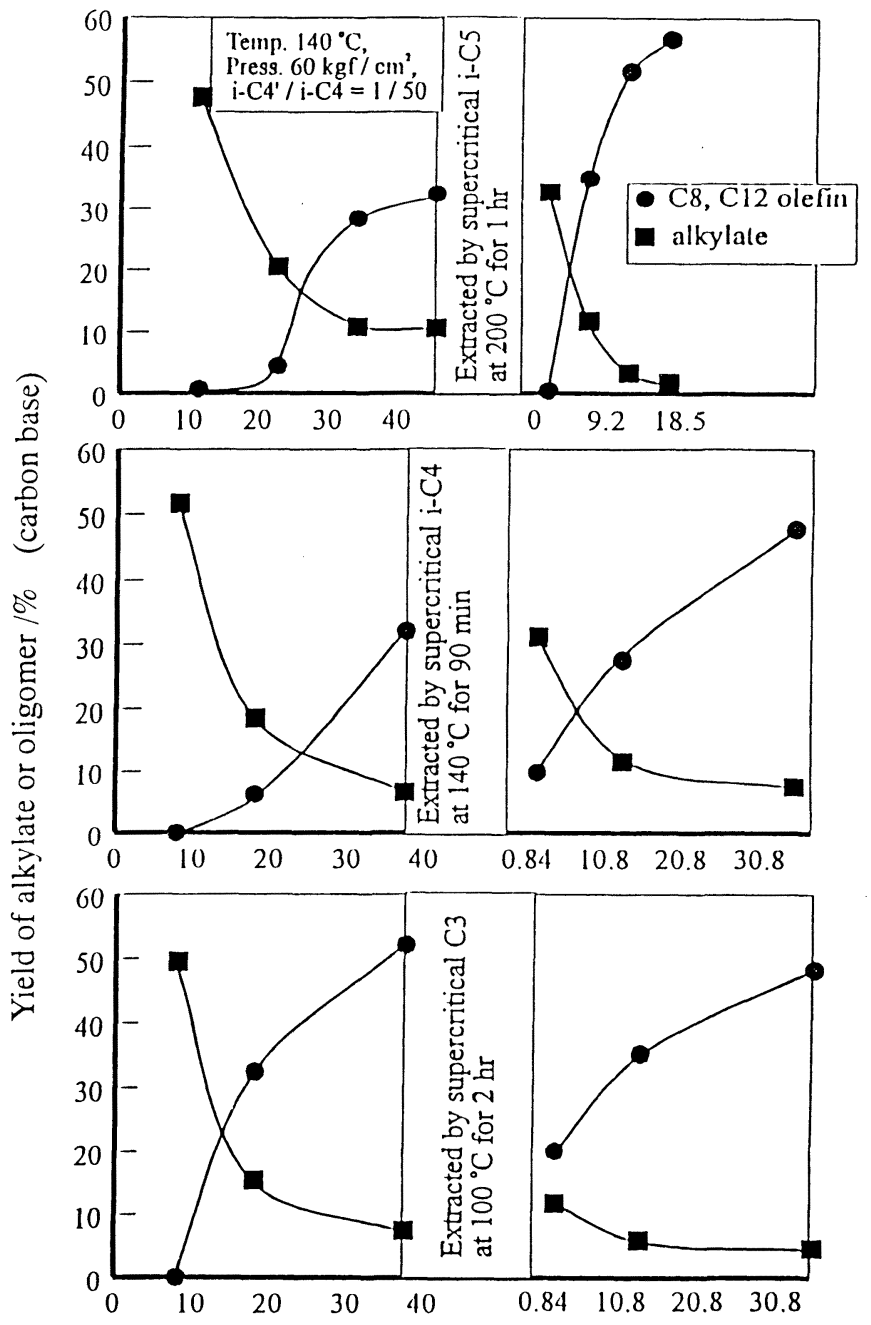

Accumulated feed amount of olefin $/ \mathrm{mmol}$ cat- $\mathrm{g}^{-1}$ Fig. 3 Extraction Effect on the Spent Catalysts 
especially at the low reaction temperature here. Low solubility of the catalyst poison into supercritical phase propane at the reaction condition deactivated catalyst.

In Fig. 3, different supercritical fluids mentioned above were used to extract the deactivated catalyst, to clarify their regeneration capacity. After 2-methyl-butane was used to extract the spent catalyst for $1 \mathrm{~h}$, the initial activity of alkylation reaction was restored to about $70 \%$ of the first initial activity. For the extraction where 2-methyl-propane was employed, similar effect was observed. But if propane was used to regenerate the deactivated catalyst, no significant result was available; regenerated catalyst still showed low alkylate yield and high oligomer yield. The results here are in good accordance to those reported in Fig. 2. It is clear that catalyst deactivation was closely related to the extraction capacity of the accompanied supercritical fluid. In situ extraction of the formed catalyst poison determined the catalyst durability. Further, failure of the extraction of these catalyst poisons improved the oligomerization reaction of olefin.

In Fig. 4, it is displayed the content of the extracted hydrocarbons by supercritical-phase 2-methyl-butane. No hydrocarbons other than $\mathrm{C} 12$ olefin (two isomers) was detected. It is clear that $\mathrm{C} 12$ olefin derived from oligomerization reaction deposited onto catalytic site and deactivated the catalyst. More concretely, highmolecule-weight olefin such as $\mathrm{C} 12$, which has high electron density, might combine strongly with Lewis acid site inside the catalyst framework, to obstruct the participation of Lewis acid into alkylation reaction. In situ extraction of $\mathrm{C} 12$ olefin from these acidic sites was critical to keep catalyst durability.

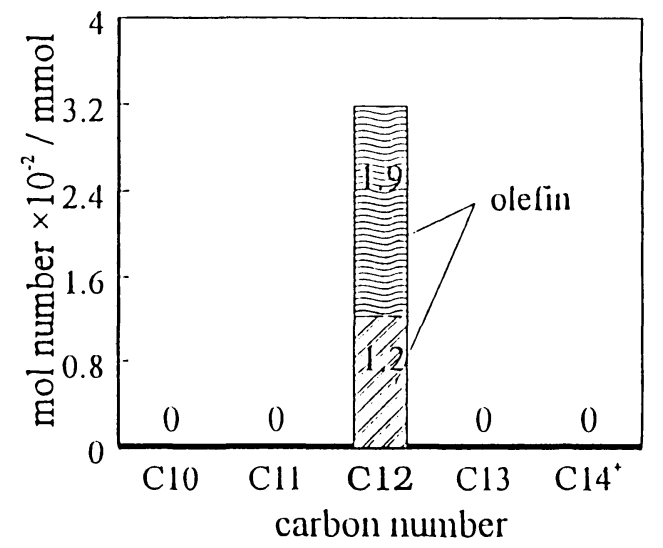

Fig. 4 Extracted Products from the Used Catalyst by Supercritical-Phase $i$-C5 at $200^{\circ} \mathrm{C}$ for $1 \mathrm{~h}$

\section{Conclusions}

The supercritical phase reaction exhibited higher activity, along with remarkably longer lifetime, on $\mathrm{Y}$ zeolite catalyst, compared to the reactions in liquid phase or gas phase, which should be attributed to the excellent extraction and transportation ability of the accompanied supercritical fluid.

\section{References}

1) Cusmano, J. A., CHEMTECH, 48, 2(1992).

2) Corma, A., Martinez, A., Catal. Rev. Sci. Eng., 35, 483(1993).

3) Savage, P. E., Gopalan, S., Brock, E., AIChE J., 41, 1723(1995).

4) Baptist-Nguyen, S., Subramaniam, B., AIC $h E$.J., 38, 1027(1992).

5) Fan, L., Yokota, K., Fujimoto, K., AIChE J., 38, 1639(1992).

6) Madras, G., Erkey, C., Akgerman, A., Ind. Eng. Chem. Res., 32, 1163(1993).

7) Nakamura, I., Ishida, S., Fujimoto, K., Preprints Div. of Petro. Chem. ACS, 40, (3), 512(1995).

\section{Keywords}

Alkylation, Zeolite, Supercritical fluid, Catalyst deactivation 